Lorentz shear modulus of fractional quantum Hall states

This article has been downloaded from IOPscience. Please scroll down to see the full text article.

2009 J. Phys.: Condens. Matter 21275603

(http://iopscience.iop.org/0953-8984/21/27/275603)

View the table of contents for this issue, or go to the journal homepage for more

Download details:

IP Address: 128.206.162.204

The article was downloaded on 14/06/2010 at $16: 54$

Please note that terms and conditions apply. 


\title{
Lorentz shear modulus of fractional quantum Hall states
}

\author{
I V Tokatly ${ }^{1,2}$ and G Vignale ${ }^{3}$ \\ ${ }^{1}$ European Theoretical Spectroscopy Facility (ETSF), Departamento de Fisica de Materiales, \\ Universidad del Pais Vasco UPV/EHU, Centro Mixto CSIC-UPV/EHU, 20018 San Sebastian, \\ Spain \\ ${ }^{2}$ Moscow Institute of Electronic Technology, Zelenograd, 124498, Russia \\ ${ }^{3}$ Department of Physics, University of Missouri-Columbia, Columbia, MO 65211, USA \\ E-mail: ilya_tokatly@ehu.es and vignaleg@missouri.edu
}

Received 24 April 2009, in final form 15 May 2009

Published 12 June 2009

Online at stacks.iop.org/JPhysCM/21/275603

\begin{abstract}
We show that the Lorentz shear modulus of macroscopically homogeneous electronic states in the lowest Landau level is proportional to the bulk modulus of an equivalent system of interacting classical particles in the thermodynamic limit. Making use of this correspondence, we calculate the Lorentz shear modulus of Laughlin's fractional quantum Hall states at filling factor $v=1 / m$ ( $m$ an odd integer) and find that it is equal to $\pm \hbar m n / 4$, where $n$ is the density of particles and the sign depends on the direction of magnetic field.
\end{abstract}

\section{Introduction}

The response of many-body systems to external fields can be universally formulated in terms of hydrodynamical equations of motion, which follow from the local conservation laws of the number of particles and momentum. These equations completely describe the dynamics of the particle density $n$ and the particle current density $\mathbf{j}$ under the combined action of external and internal (or stress) forces. The most important ingredient of this formulation is the stress tensor $P_{i k}$, which determines the stress force density entering the momentum conservation law: $F_{i}^{\text {stress }}=-\partial_{k} P_{i k}$. In general the stress tensor for every particular state of matter is a universal functional of the current density [1] (or, equivalently, of the deformation tensor [2, 3]). In fact, the form of $P_{i k}[\mathbf{j}]$ encodes all the dynamical information about a given many-body state.

Unfortunately it is an extremely difficult, if not hopeless task to determine the general form of the stress tensor as a functional of the current. As usual the problem simplifies in the linear response regime as the stress tensor becomes a linear functional of the strain tensor $u_{i k}=\frac{1}{2}\left(\partial_{i} u_{k}+\partial_{k} u_{i}\right)$, where $\mathbf{u}(\mathbf{r}, \omega)$ is the displacement vector field. The coefficients of this linear functional form the rank-4 tensor of elasticity $Q_{i j k l}(\omega)$, which is, in general, a function of frequency. This tensor describes a stress response to a time dependent deformation of the system. In a macroscopically isotropic two-dimensional (2D) system subjected to a perpendicular magnetic field the rank-4 tensor $Q_{i j k l}$ contains only three independent components and can be parametrized by three dynamic 'elastic moduli' $K(\omega), \mu(\omega)$, and $\Lambda(\omega)$ [4-7]. The moduli $K(\omega)$ and $\mu(\omega)$ describe the response to a local change in volume and to a local volume-preserving (shear) deformation, respectively. Therefore they correspond to the standard bulk and shear moduli of classical elasticity theory [8]. The third modulus, $\Lambda(\omega)$, appears only in the presence of the magnetic field and plays an important role in the dynamics of 2D electrons at high magnetic field. This modulus controls the magnitude of a stress proportional to the rate of volume-preserving deformations, and in this respect is similar to a viscous stress. However, the corresponding force always acts in a direction perpendicular to the stream lines and is purely nondissipative, like the usual Lorentz force. To underline its nondissipative character, in [5-7] we named the modulus $\Lambda(\omega)$ 'Lorentz shear modulus'. The same quantity is also known in the literature as 'asymmetric viscosity' [9], or 'Hall viscosity' [10].

The appearance of a 'nondissipative viscosity' in the presence of a magnetic field is well known in plasma physics. The calculation of the corresponding kinetic coefficient $\Lambda_{0}=$ - $\lim _{\omega \rightarrow 0} \Lambda(\omega)$ for a classical plasma can be found, for example, in [11]. The result is

$$
\Lambda_{0}= \pm \frac{\hbar n}{2} \frac{k_{\mathrm{B}} T}{\hbar \omega_{\mathrm{c}}}
$$

where $n$ is the electronic density, $T$ is the temperature and $\omega_{\mathrm{c}}$ is the cyclotron frequency (the sign is determined by the direction of the magnetic field). 
In this paper we focus on the microscopic calculation of $\Lambda_{0}$ for an extended, macroscopically homogeneous $2 \mathrm{D}$ electronic system in the quantum Hall regime, i.e. when all the electrons reside in the lowest Landau level. For a 2D noninteracting electron liquid in a completely filled Landau level, this calculation was first done by Avron et al in [9]. These authors observed that the value of $\Lambda_{0}$ is proportional to a Berry curvature related to adiabatic changes of geometry, and used this fact to explicitly calculate the modulus. The result was $\Lambda_{0}= \pm \hbar n / 4,{ }^{4}$ which, incidentally, agrees with the classical formula (1) if one replaces the thermal kinetic energy per particle $k_{\mathrm{B}} T$ with the kinetic energy per particle of the lowest Landau level $\hbar \omega_{\mathrm{c}} / 2$.

In [4] one of us derived hydrodynamics equations for quantum Hall states at fractional filling factor using a fermionic Chern-Simons theory [12-15] at the RPA level. Within this approximation $\Lambda_{0}$ exactly coincides with the noninteracting result of [9], as reported above, which is not surprising since the composite fermions do not interact in RPA. In a recent paper [7] we applied a formally exact linear response theory to the calculation of the Lorentz shear modulus for strongly correlated quantum Hall states at fractional filling factors (Laughlin states). We showed that $\Lambda(\omega)$ can be expressed in terms of a particular stress-stress correlation function which, in the limit $\omega \rightarrow 0$, reduces to the Berry curvature expression of Avron et al. After publication of our work [7] the problem of calculating $\Lambda_{0}$ was reconsidered by Read [10] who noticed an error at the very end of our calculation. Making use of the Laughlin plasma analogy [16] Read calculated the Hall viscosity (Lorentz shear modulus) for Laughlin states at $v=1 / m$ ( $m$ is an odd integer) and also for states with trial wavefunctions in the form of conformal blocks of a conformal field theory. For Laughlin states, Read's result is

$$
\Lambda_{0}= \pm \frac{\hbar n m}{4}
$$

whereas in our paper we had incorrectly obtained $\Lambda_{0}= \pm \frac{\hbar n}{4}$.

Read's paper is difficult and contains much more than just the calculation of the Lorentz shear modulus. We have found that it is possible to give a more elementary derivation of Read's result for filling factors $v=1 / m$. The crucial step in the derivation is the recognition that the calculation of the Lorentz shear modulus for a macroscopically homogeneous state of electrons in the lowest Landau level can be mapped to the calculation of the bulk modulus of an equivalent system of interacting classical particles. While in general the many-body interactions in this equivalent classical system are prohibitively complicated, they simplify dramatically for Laughlin's quantum Hall states, where one recovers the wellknown classical plasma analogy. This allows a simple calculation of the Lorentz shear modulus. The quantumclassical correspondence can also be used 'in reverse'. Namely, from the knowledge of the shear modulus associated with a certain wavefunction in the lowest Landau level one can in principle obtain the bulk modulus of the equivalent classical system, even if the latter is very complicated.

${ }^{4}$ Actually the original paper [9] missed a factor 2 resulting in one half the correct value quoted in the main text.
The structure of this paper is as follows. In section 2.1 we introduce the formal definition of the Lorentz shear modulus and discuss its physical significance. Here we also address the delicate question of the correct boundary conditions for the trial many-body wavefunction to be used in the calculation of the Berry curvature in the thermodynamic limit. The calculation of the Lorentz shear modulus for the Laughlin states is presented in section 2.2. In section 2.3 we generalize our procedure to other macroscopically homogeneous states in the lowest Landau level. In section 3 we summarize our main results and discuss how the new value of $\Lambda_{0}$ affects our previous analysis of the collective modes of the electron liquid in the fractional quantum Hall regime.

\section{Lorentz shear modulus in a 2D magnetized electron gas}

\subsection{Definitions and physical significance of the Lorentz shear modulus}

Let us consider a 2D electron gas confined to the $(x, y)$ plane and subjected to a perpendicular magnetic field $\mathbf{B}=B \hat{\mathbf{z}}$. In the linear response regime and in the long wavelength limit the exact stress tensor $P_{i j}$ takes the following 'elastic' form $[6,7]$

$$
P_{i j}(\mathbf{r}, \omega)=-Q_{i j k l}(\omega) u_{k l}(\mathbf{r}, \omega),
$$

where $u_{k l}=\frac{1}{2}\left(\partial_{k} u_{l}+\partial_{l} u_{k}\right)$ is the strain tensor and $\mathbf{u}$ is the displacement vector defined in the standard way, namely $\partial_{t} \mathbf{u}=\mathbf{j} / n=\mathbf{v}$ is the velocity field of the electron liquid ( $\mathbf{j}$ is the current density). The frequency dependent coefficients $Q_{i j k l}(\omega)$ in the linear functional of equation (3) form the dynamic tensor of elasticity. The general structure of this tensor is essentially fixed by symmetry. In particular, for a macroscopically isotropic state the rank-4 tensor $Q_{i j k l}$ is completely determined by only three independent 'elastic moduli'

$$
\begin{gathered}
Q_{i j k l}(\omega)=K(\omega) \delta_{i j} \delta_{k l}+\mu(\omega)\left(\delta_{i k} \delta_{j l}+\delta_{i l} \delta_{j k}-\delta_{i j} \delta_{k l}\right) \\
+\mathrm{i} \omega \frac{\Lambda(\omega)}{2}\left(\varepsilon_{i k} \delta_{j l}+\varepsilon_{j k} \delta_{i l}+\varepsilon_{i l} \delta_{j k}+\varepsilon_{j l} \delta_{i k}\right) .
\end{gathered}
$$

Inserting equation (3) into (4) we get the following representation for the stress tensor for any isotropic state of a 2D magnetized electron gas

$$
\begin{aligned}
P_{i j} & =-K \delta_{i j} u_{k k}-\mu\left(2 u_{i j}-\delta_{i j} u_{k k}\right) \\
& +\Lambda\left(\varepsilon_{i k} v_{k j}+\varepsilon_{j k} v_{k i}\right),
\end{aligned}
$$

where $v_{i j}=-\mathrm{i} \omega u_{i j}=\frac{1}{2}\left(\partial_{i} v_{j}+\partial_{j} v_{i}\right)$ is the rate of change of the strain. The first two terms in equation (5) describe the stress arising from a local change in volume $\left(u_{k k}=\nabla \mathbf{u}\right)$ and from a volume-preserving (traceless, or shear) deformation $\left(2 u_{i j}-\right.$ $\left.\delta_{i j} u_{k k}\right)$, respectively. Hence the corresponding coefficients $K(\omega)$ and $\mu(\omega)$ have the same meaning as the standard bulk and shear moduli of a homogeneous elastic medium [8]. The third term in equation (5) is proportional to the rate of change of the strain, i.e. to velocity gradients, which looks somewhat similar to a viscous stress. However, for real $\Lambda$ this term is time-reversal invariant and, therefore, does not cause dissipation. To get a more intuitive picture we consider a 'shear 


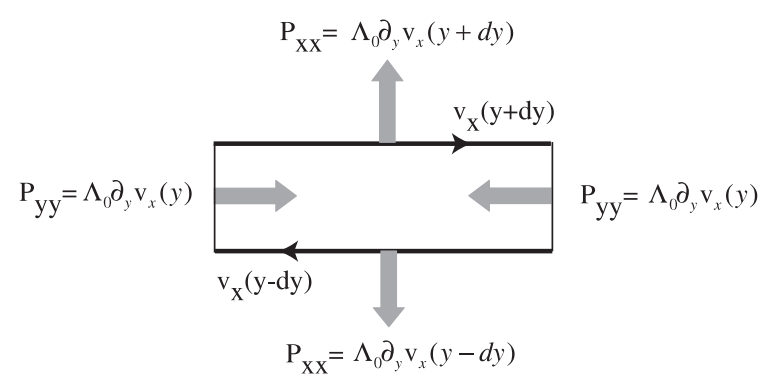

Figure 1. Lorentz shear forces acting on a small rectangular element of the electron fluid according to equation (6).

flow' with the velocity distribution of the form $\mathbf{v}=\left(v_{x}(y), 0\right)$ (for example, two oppositely directed streamlines). In this simple case the $\Lambda$-part of the stress tensor has only two nonzero elements,

$$
P_{x x}^{\Lambda}=-P_{y y}^{\Lambda}=\Lambda \partial_{y} v_{x}(y) .
$$

This distribution of stresses corresponds to forces, exerted on the faces of an infinitesimal rectangle, which squeeze or repel (depending on the sign of $\Lambda$ ) two opposite stream lines. The important point is that these forces are always perpendicular to the local direction of the streamlines, as shown in figure 1. Hence the effect of the third term in equation (5) is analogous to that of the Lorentz (Ampere) force acting between two currents, and causes no dissipation. Having in mind this physical picture we prefer to call the coefficient $\Lambda(\omega)$ the 'Lorentz shear modulus' in contrast to more formal terms such as 'asymmetric viscosity' [9] or 'Hall viscosity' [10].

\subsection{Linear response approach to the microscopic calculation of the Lorentz shear modulus}

Let us now address the problem of the microscopic calculation of the Lorentz shear modulus in the limit of zero frequency. In our recent paper [7] we expressed $\Lambda_{0} \equiv-\lim _{\omega \rightarrow 0} \Lambda(\omega)$ in terms of the stress-stress correlation function in the following manner:

$$
\Lambda_{0}=-\lim _{\omega \rightarrow 0} \lim _{\mathbf{q} \rightarrow 0} \frac{1}{\omega} \operatorname{Im}\left\langle\left\langle\hat{P}_{x x} ; \hat{P}_{x y}\right\rangle\right\rangle_{\mathbf{q}, \omega}
$$

The stress tensor operator $\hat{P}_{i j}$ is formally defined as follows (see, e. g. [3])

$$
\hat{P}_{i j}(\mathbf{r}, t)=-2\left[\frac{\delta \hat{H}\left[g_{i j}\right]}{\delta g_{i j}(\mathbf{r}, t)}\right]_{g_{i j}=\delta_{i j}},
$$

where $\hat{H}\left[g_{i j}\right]$ is the Hamiltonian of the system in a 'deformed' space with metric $g_{i j}(\mathbf{r}, t)$. The subscript $\mathbf{q}$ in equation (7) means that we are actually considering the correlation function for the Fourier component of $\hat{P}_{i j}(\mathbf{r}, t)$ at wavevector $\mathbf{q}$, where $\mathbf{q}$ tends to zero before $\omega$. It is convenient to parametrize the metric tensor as follows [9, 17, 7]

$$
g_{i j}=\frac{J}{\tau_{2}}\left(\begin{array}{cc}
1 & \tau_{1} \\
\tau_{1} & |\tau|^{2}
\end{array}\right)
$$

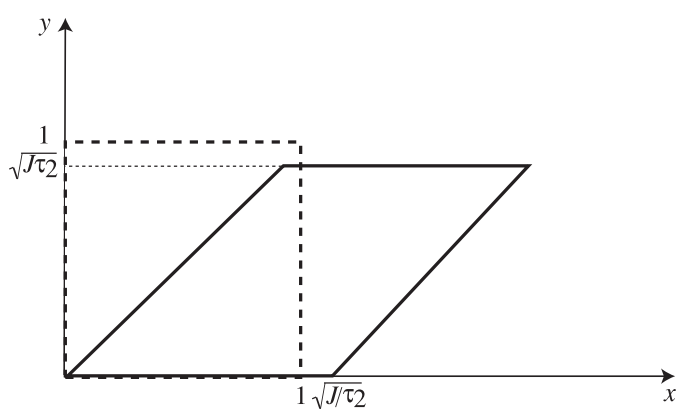

Figure 2. Deformation of the Euclidean plane corresponding to the metric of equation (9). The square is transformed into a parallelogram. The slope of the oblique side coincides with the direction of $\tau$ in the complex plane. We have chosen $J=1$, $\tau_{1}=\tau_{2}<1$.

where $\tau=\tau_{1}+i \tau_{2}$. A picture of the deformation of the Euclidean plane corresponding to this choice of metrics is shown in figure 2. Making use of the Lehmann representation [18] for the stress-stress correlation function and performing the standard manipulations one can transform equation (7) to the following Berry curvature form

$$
\Lambda_{0}=\frac{2 \hbar}{L^{2}} \operatorname{Im}\left\langle\frac{\partial \Psi_{0}}{\partial \tau_{1}} \mid \frac{\partial \Psi_{0}}{\partial \tau_{2}}\right\rangle
$$

where $L^{2}$ is the area of the system, $\Psi_{0}$ is the ground state wavefunction in a homogeneously deformed space with a constant metric of equation (9), and the $\tau$-derivatives are calculated at $\tau_{1}=0, \tau_{2}=1$, and $J=1$.

Equation (10) was first derived in [9] from the adiabatic response theory. Our linear response derivation shows that there is a delicate point in the identification of the Berry curvature (the right-hand side in equation (10)) with the physical Lorentz shear modulus. Physically such a modulus describes a stress response to a deformation whose wavelength is much larger than any internal scale of the system (e.g. the interparticle distance, the correlation length, etc), but still much smaller than the size of the sample $L$. At the level of the linear response formula, equation (7), this means that the thermodynamic limit should be performed before the limit $\mathbf{q} \rightarrow 0$. This guarantees that the calculated elastic modulus does not depend on the geometry of the sample (provided the sample is sufficiently large), i.e. it is a bulk property of the 'material', as it should. However, in the derivation of the Berry curvature formula the order of limits was explicitly interchanged. The right-hand side of equation (10) contains the ground state wavefunction of a finite system with a finite number of particles $N$. Physically this describes the response to a homogeneous deformation of the whole sample which in general may depend on the sample geometry ${ }^{5}$. Nonetheless it

5 A similar situation arises in the standard textbook calculation of the plasma frequency using a finite slab of jellium (see, for example [23]). When the electrons are uniformly displaced relative to the slab, a simple calculation of the restoring force yields the plasma frequency $\omega_{\mathrm{p}}=\sqrt{4 \pi n e^{2} / m}$, which coincides with the $\mathbf{q} \rightarrow 0$ limit of the bulk plasmon mode. If, however, one does the same calculation using a finite jellium of spherical shape, one gets a different result $\omega_{\mathrm{p}}^{\prime}=\sqrt{4 \pi n e^{2} / 3 m}$, which does not coincide with the $\mathbf{q} \rightarrow 0$ limit of the bulk plasmon. The essential point is that the bulk plasmon is a plane wave excitation, which in the $\mathbf{q} \rightarrow 0$ limit $(q \ll 1 / L)$ goes smoothly over to the slab model, not to the spherical jellium model. 
is still possible to obtain the correct physical result even with the interchanged order of limits. The key is to use boundary conditions for the wavefunction $\Psi_{0}$, which are compatible with the symmetry of the physical plane wave perturbation (the deformation in our particular case). For, in this way we ensure that the $\mathbf{q} \rightarrow 0$ limit of the deformed state goes smoothly to the homogeneous deformation of the ground state in the same geometry. In practice this simply means that the correct result is guaranteed if one studies a sample of rectangular shape, which satisfies periodic boundary conditions either in two directions (torus) or in one direction (cylinder). On the other hand a sample of circular shape would not be compatible with the symmetry of the plane wave, because the presence of a finite wavevector, no matter how small, always breaks rotational symmetry ${ }^{6}$.

The choice of the torus geometry in the present context may also cause some technical problems which require special care. On a torus the ground state for gapped quantum Hall states is degenerate. This nicely demonstrates the topological nature of these states, but at the same time introduces an artificial degeneracy which is not present in any real physical sample. Clearly, this degeneracy should not affect the physical elastic moduli. To guarantee that this is indeed the case we put our system of $N$ electrons on a cylinder with a circumference $L$ in such a way that it occupies the area $L^{2}=N_{\mathrm{S}}\left(2 \pi \ell^{2}\right)$, where $N_{\mathrm{S}}=N / v$ is the number of flux quanta (one flux quantum $=h c / e$ ), and $\ell=\sqrt{\hbar c / e B}$ is the magnetic length. The thermodynamic limit which we take at the end of calculations corresponds to $N \rightarrow \infty$ at fixed filling factor $v=N / N_{\mathrm{S}}$.

For the Hamiltonian defined on a space with the metric of equation (9) the $N$-body wavefunction which lies entirely in the lowest Landau level and satisfies periodic boundary conditions in the $x$-direction can be written in the following general form

$$
\Psi_{0}\left(\mathbf{r}_{1}, \ldots, \mathbf{r}_{N}\right)=Z^{-\frac{1}{2}}\left(\tau_{1}, \tau_{2}\right) f\left(\eta_{1}, \ldots, \eta_{N}\right) \prod_{i=1}^{N} \mathrm{e}^{\frac{\mathrm{i}}{2 \ell^{2}} \tau y_{i}^{2}}
$$

where $Z^{-\frac{1}{2}}$ is the normalization factor, $\eta_{j} \equiv \exp \left(2 \pi \mathrm{i} \frac{x_{j}+\tau y_{j}}{L}\right)$, and $f\left(\eta_{1}, \ldots, \eta_{N}\right)$ is an analytic function of its arguments. A crucial observation is that $\Psi_{0}$, equation (11), apart from the normalization factor, is an analytic function of the complex variable $\tau$. This enables us to express the Berry curvature solely in terms of the normalization factor $[17,7]$ :

$$
2 \operatorname{Im}\left\langle\frac{\partial \Psi_{0}}{\partial \tau_{1}} \mid \frac{\partial \Psi_{0}}{\partial \tau_{2}}\right\rangle=\frac{1}{2}\left(\frac{\partial^{2}}{\partial \tau_{1}^{2}}+\frac{\partial^{2}}{\partial \tau_{2}^{2}}\right) \ln Z .
$$

Hence the problem of microscopic calculation of the Lorentz shear modulus reduces to the calculation of the normalization factor. For our particular choice of a quantum Hall system on a cylinder the problem simplifies even further since the normalization factor depends only on $\tau_{2}$. Indeed, by shifting

6 In fact, one can show that the original Laughlin wavefunction for a 'circular droplet' [16], when used in equation (10), yields a result that is vastly different from the one obtained from Laughlin states on a torus [24] or on a cylinder [19]. all the $x$-variables, $\left(x_{j}+\tau_{1} y_{j}\right) \rightarrow x_{j}$, and rescaling all $y$ variables, $\tau_{2} y_{j} \rightarrow y_{j}$, we reduce the normalization integral to the following form

$$
\begin{aligned}
Z\left(\tau_{2}\right) & =\tau_{2}^{-N} \prod_{k=1}^{N} \int_{0}^{L} \mathrm{~d} x_{k} \int_{-\infty}^{\infty} \mathrm{d} y_{k} \\
& \times\left|f\left(\mathrm{e}^{2 \pi \mathrm{i} z_{1} / L}, \ldots, \mathrm{e}^{2 \pi \mathrm{i} z_{N} / L}\right)\right|^{2} \mathrm{e}^{-\sum_{j=1}^{N} \frac{y_{j}^{2}}{\tau_{2} \ell^{2}}},
\end{aligned}
$$

where $z_{k}=x_{k}+\mathrm{i} y_{k}$. Hence the final formula for the Lorentz shear modulus in the state $\Psi_{0}$ takes the form

$$
\Lambda_{0}=\frac{\hbar}{2 L^{2}}\left[\frac{\partial^{2}}{\partial \tau_{2}^{2}} \ln Z\left(\tau_{2}\right)\right]_{\tau_{2}=1}
$$

with $Z\left(\tau_{2}\right)$ defined after equation (13). In the next subsections we calculate this integral using the Laughlin classical plasma analogy.

\subsection{Calculation of the Lorentz shear modulus for Laughlin states}

The cylindrical generalization of the Laughlin trial function at $v=1 / m$ contains the analytic factor $f$ of the following form [19]

$$
f\left(z_{1}, \ldots, z_{N}\right)=\prod_{j<k}\left(\mathrm{e}^{2 \pi \mathrm{i} z_{j} / L}-\mathrm{e}^{2 \pi \mathrm{i} z_{k} / L}\right)^{m} .
$$

Inserting this equation into equation (13) we represent the normalization integral in a form of a partition function of $N$ classical particles,

$$
Z\left(\tau_{2}\right)=\tau_{2}^{-N} \prod_{k=1}^{N} \int_{0}^{L} \mathrm{~d} x_{k} \int_{-\infty}^{\infty} \mathrm{d} y_{k} \mathrm{e}^{-\frac{1}{v} W\left(\mathbf{r}_{1}, \ldots, \mathbf{r}_{N}\right)},
$$

at the 'temperature' $v=1 / m$ and with the following energy

$$
W\left(\mathbf{r}_{1}, \ldots, \mathbf{r}_{N}\right)=\frac{2 \pi N}{\tau_{2} L^{2}} \sum_{j=1}^{N} y_{j}^{2}+\frac{1}{2} \sum_{j \neq k} \tilde{V}\left(\mathbf{r}_{j}, \mathbf{r}_{k}\right),
$$

where we used the identity $1 / m=N / N_{\mathrm{S}} \equiv 2 \pi N \ell^{2} / L^{2}$, and introduced the notation

$$
\tilde{V}\left(\mathbf{r}, \mathbf{r}^{\prime}\right)=-\ln \left|\mathrm{e}^{2 \pi \mathrm{i} z_{j} / L}-\mathrm{e}^{2 \pi \mathrm{i} z_{k} / L}\right|^{2} .
$$

The first term in equation (17) has a clear physical interpretation; it is the Coulomb potential of a homogeneously distributed (on the cylindrical surface) positive charge with density $\rho=N /\left(\tau_{2} L^{2}\right)$. Note that after the rescaling of the $y$ coordinate the size of the system along the axis of the cylinder becomes $L_{y}=\tau_{2} L$; the classical system with the probability distribution $\sim \exp \left[-m W\left(\left\{\mathbf{r}_{j}\right\}\right)\right]$ occupies the region with $0<$ $y<\tau_{2} L$. Hence the quantity $N /\left(\tau_{2} L^{2}\right)$ entering the first term in equation (17) exactly coincides with the physical density of this classical system, in perfect agreement with the idea of the Laughlin classical plasma analogy [16]. However, at variance with the original circularly symmetric Laughlin wavefunction, the second term in equation (17) does not look like the interaction energy of a Coulomb plasma. To show 
that this is nonetheless the case, we express the two-point 'potential' $\tilde{V}\left(\mathbf{r}, \mathbf{r}^{\prime}\right)$, equation (18), in terms of the physical Coulomb interaction $V\left(\mathbf{r}-\mathbf{r}^{\prime}\right)$ between two-point particles on a cylinder, (see appendix)

$$
V\left(\mathbf{r}-\mathbf{r}^{\prime}\right)=-2 \pi \frac{\left|y-y^{\prime}\right|}{L}-\ln \left|1-\mathrm{e}^{2 \pi \mathrm{i} \frac{x-x^{\prime}+\mathrm{i}\left|y-y^{\prime}\right|}{L}}\right|^{2} .
$$

The identity of equation (A.4) allows us to relate $\tilde{V}\left(\mathbf{r}, \mathbf{r}^{\prime}\right)$ to $V\left(\mathbf{r}-\mathbf{r}^{\prime}\right)$

$$
\tilde{V}\left(\mathbf{r}, \mathbf{r}^{\prime}\right)=2 \pi \frac{y+y^{\prime}}{L}+V\left(\mathbf{r}-\mathbf{r}^{\prime}\right)
$$

Substituting this equation into equation (17) we transform the energy $W\left(\left\{\mathbf{r}_{j}\right\}\right)$ to the following form

$W\left(\left\{\mathbf{r}_{j}\right\}\right)=-\tau_{2} \frac{\pi}{2}(N-1)^{2}+\frac{2 \pi N}{\tau_{2} L^{2}} \sum_{j=1}^{N} \tilde{y}_{j}^{2}+\frac{1}{2} \sum_{j \neq k} V\left(\tilde{\mathbf{r}}_{j}-\tilde{\mathbf{r}}_{k}\right)$,

where $\tilde{x}_{k}=x_{k}$ and $\tilde{y}_{k}=y_{k}+\frac{1}{2} \tau_{2} L\left(1-\frac{1}{N}\right)$. Hence the main effect of the difference between $\tilde{V}\left(\mathbf{r}, \mathbf{r}^{\prime}\right)$ and $V\left(\mathbf{r}-\mathbf{r}^{\prime}\right)$ is a shift of the $y$-coordinate by a half of the system size $\sim \tau_{2} L / 2$. In what follows we remove this shift by moving the origin to the center of the slab: $\tilde{y}_{k} \rightarrow y_{k}$.

Thus the energy $W\left(\left\{\mathbf{r}_{j}\right\}\right)$ exactly corresponds to the energy of a system of $N$ classical charges on an infinite homogeneously charged cylinder. To proceed further in the calculation of the partition function of this system we separate a 'neutralizing' part of the background with density

$$
n_{\mathrm{b}}(\mathbf{r})=\frac{N}{\tau_{2} L^{2}} \theta\left(\tau_{2} \frac{L}{2}-|y|\right),
$$

and total charge $\int \mathrm{d} \mathbf{r} n_{\mathrm{b}}(\mathbf{r})=N$. The next step is to separate the energy of interaction of the particles with $n_{\mathrm{b}}(\mathbf{r})$ from the total energy of equation (21). Using equations (A.5) and (A.6) of the appendix we find the following representation for $W\left(\left\{\mathbf{r}_{j}\right\}\right)$

$$
W=-\tau_{2} \pi\left(\frac{2 N^{2}}{3}-N+\frac{1}{2}\right)+E_{\mathrm{NJ}}\left(\left\{\mathbf{r}_{j}\right\}\right)+E_{\mathrm{S}}\left(\left\{\mathbf{r}_{j}\right\}\right) .
$$

The second term, $E_{\mathrm{NJ}}\left(\left\{\mathbf{r}_{j}\right\}\right)$, in this equation is the energy of a neutral slab of jellium,

$$
E_{\mathrm{NJ}}=\frac{1}{2} \int \mathrm{d} \mathbf{r} \mathrm{d} \mathbf{r}^{\prime} V\left(\mathbf{r}-\mathbf{r}^{\prime}\right)\left[\Delta \hat{\rho}(\mathbf{r}) \Delta \hat{\rho}\left(\mathbf{r}^{\prime}\right)-\delta\left(\mathbf{r}-\mathbf{r}^{\prime}\right) \hat{\rho}(\mathbf{r})\right]
$$

where $\hat{\rho}(\mathbf{r})$ is the microscopic particle density, and $\Delta \hat{\rho}(\mathbf{r})$ is the microscopic charge density of jellium:

$$
\hat{\rho}(\mathbf{r})=\sum_{j=1}^{N} \delta\left(\mathbf{r}-\mathbf{r}_{j}\right), \quad \Delta \hat{\rho}(\mathbf{r})=\hat{\rho}(\mathbf{r})-n_{\mathrm{b}}(\mathbf{r}) .
$$

The third term in equation (23) corresponds to the surface (edge) contribution

$$
E_{\mathrm{S}}=\frac{2 \pi N}{\tau_{2} L^{2}} \sum_{j=1}^{N}\left(\left|y_{j}\right|-\frac{\tau_{2} L}{2}\right)^{2} \theta\left(\left|y_{j}\right|-\frac{\tau_{2} L}{2}\right),
$$

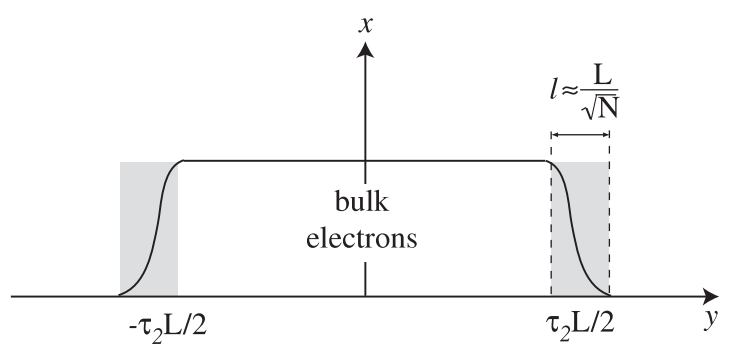

Figure 3. Schematic sketch of the electron density as a function of $y$. The two edge regions, whose width is proportional to the mean interparticle distance $\ell \sim \frac{L}{\sqrt{N}}$, are highlighted. The actual structure of the edge is more complicated [20], and is not shown here.

which is the potential energy of particles outside the 'neutralizing' part of the background (see figure 3).

Equation (23) is extremely useful for the analysis of the relevant limit $N \rightarrow \infty$ of the partition function. It is physically obvious that the contribution, equation (26), should be irrelevant in the thermodynamic limit as it involves a small fraction of particles in the edge region. Indeed, from figure 3 we see that the contribution to $E_{\mathrm{S}}$ comes from a region whose width is of the order of the mean interparticle distance $\ell \sim$ $\frac{1}{\sqrt{n}}=\frac{L}{\sqrt{N}}$ (since it is proportional to the magnetic length, we use the same notation). Then a simple, order of magnitude estimate of $E_{\mathrm{S}}$ is

$$
E_{\mathrm{S}} \sim \frac{N}{L^{2}}(L \ell n) \ell^{2} \sim \sqrt{N}
$$

where $L \ell n \sim \sqrt{N}$ is the average number of particles in the edge region. This shows that in the limit $N \rightarrow \infty E_{\mathrm{S}}$ grows as $\sqrt{N}$ and is negligible in comparison with the first and the second terms in equation (23), which are proportional to $N^{2}$ and $N$ respectively. Inserting the energy of equation (23) into (16) and neglecting $E_{\mathrm{S}}$, we rewrite the normalization integral as follows

$$
Z\left(\tau_{2}\right)=L^{2 N} \mathrm{e}^{m \tau_{2} \pi\left(\frac{2 N^{2}}{3}-N+\frac{1}{2}\right)} \frac{Z_{\mathrm{NJ}}}{Z_{0}},
$$

where $Z_{\mathrm{NJ}}=\left\langle\exp \left[-m E_{\mathrm{NJ}}\left(\left\{\mathbf{r}_{j}\right\}\right)\right]\right\rangle$ is the partition function of the slab of jellium, and $Z_{0}=\left(\tau_{2} L^{2}\right)^{N}$ is the partition function of $N$ noninteracting particles in the slab. Hence equation (28) can be equivalently represented in the form

$$
Z\left(\tau_{2}\right)=L^{2 N} \mathrm{e}^{m \tau_{2} \pi\left(\frac{2 N^{2}}{3}-N+\frac{1}{2}\right)} \mathrm{e}^{-m F_{\text {int }}\left(\tau_{2}\right)},
$$

where $F_{\text {int }}=-\frac{1}{m} \ln \left(Z_{\mathrm{NJ}} / Z_{0}\right)$ is the interaction free energy of the classical jellium model. The final step is to substitute equation (29) into the expression of equation (14) for the Lorentz shear modulus. The first, non-extensive exponent $\left(\sim N^{2}\right)$ in equation (29) does not contribute to $\Lambda_{0}$ since it is linear in $\tau_{2}$. Therefore equation (14) reduces to the form

$$
\Lambda_{0}=-\frac{\hbar m}{2}\left[\frac{\partial^{2}}{\partial \tau_{2}^{2}} \tau_{2} \frac{F_{\text {int }}\left(\tau_{2}\right)}{\tau_{2} L^{2}}\right]_{\tau_{2}=1} .
$$

Now we can easily perform the thermodynamic limit. In this limit, the free energy density of a neutral jellium depends only 
on the density of particles, which is equal to the background charge density $\rho$ of the jellium $F_{\mathrm{NJ}} /\left(\tau_{2} L^{2}\right)=f_{\mathrm{NJ}}(\rho)$, where $\rho=n / \tau_{2}$ and $n=N / L^{2}$ is the density of electrons in the original quantum Hall system. Thus in the thermodynamic limit equation (30) simplifies as follows

$$
\Lambda_{0}=-\frac{\hbar m}{2}\left[\frac{\partial^{2}}{\partial \tau_{2}^{2}} \tau_{2} f_{\text {int }}\left(\frac{n}{\tau_{2}}\right)\right]_{\tau_{2}=1},
$$

where $f_{\text {int }}(\rho)$ is the interaction free energy density of a 2D classical jellium model with logarithmic interaction between the particles. The key observation is that $\tau_{2}$ enters equation (31) as an effective volume (the area in 2D). Hence the second derivative of the energy with respect to $\tau_{2}$ in equation (31) is nothing but the isothermal bulk modulus:

$$
\Lambda_{0}=\frac{\hbar m}{2}\left[\frac{\partial}{\partial \tau_{2}} P_{\text {int }}\left(\frac{n}{\tau_{2}}\right)\right]_{\tau_{2}=1}=-\frac{\hbar m}{2} K_{\text {int }}(n),
$$

where $P_{\text {int }}(\rho)$ and $K_{\text {int }}(\rho)$ are the interaction contributions to the pressure and bulk modulus, respectively. Equation (32) is the main result of the present paper. It shows that the calculation of the Lorentz shear modulus in a quantum Hall system reduces to the calculation of the bulk modulus of an equivalent classical system. In section 2.4 we will demonstrate that this result holds more generally for any macroscopically homogeneous quantum Hall state.

For the Laughlin states considered in this subsection we need the bulk modulus $K_{\text {int }}(\rho)$ of a 2D Coulomb plasma with logarithmic interaction, which can be calculated exactly [21]. The easiest way is to use the virial representation for the interaction pressure

$P_{\text {int }}=-\frac{1}{4 S} \int \mathrm{d} \mathbf{r} \mathrm{d} \mathbf{r}^{\prime}\left(\mathbf{r}-\mathbf{r}^{\prime}\right) \frac{\partial V\left(\mathbf{r}-\mathbf{r}^{\prime}\right)}{\partial\left(\mathbf{r}-\mathbf{r}^{\prime}\right)} \rho(\mathbf{r}) \rho\left(\mathbf{r}^{\prime}\right)\left[g\left(\mathbf{r}, \mathbf{r}^{\prime}\right)-1\right]$

where $S$ is the area of the system and $g\left(\mathbf{r}, \mathbf{r}^{\prime}\right)$ is the pair correlation function. Inserting $V(\mathbf{r})=-\ln |\mathbf{r}|^{2}$ into equation (33) and using the sum rule

$$
\int \mathrm{d} \mathbf{r}^{\prime} \rho\left(\mathbf{r}^{\prime}\right)\left[g\left(\mathbf{r}, \mathbf{r}^{\prime}\right)-1\right]=-1
$$

we obtain the interaction pressure and the corresponding bulk modulus in the form

$$
P_{\text {int }}(\rho)=K_{\text {int }}(\rho)=-\frac{1}{2} \rho .
$$

This implies the following final result for the Lorenz shear modulus of the Laughlin quantum Hall liquids

$$
\Lambda_{0}=\frac{\hbar n m}{4}=\frac{\pi \hbar}{2 \ell^{2}}
$$

which is in agreement with the recent result of Read [10]. In the case of a full Landau level $(m=1)$ it recovers the formula for the 'asymmetric viscosity' obtained by Avron et al in [9]. It is interesting to note a surprising feature of equation (34): because the density $n$ is proportional to $1 / m$ the Lorentz shear modulus for Laughlin states does not depend on the filling factor.

\subsection{Generalization to arbitrary macroscopically homogeneous quantum Hall states}

The result of equation (32) can be straightforwardly generalized to other macroscopically homogeneous states in the lowest Landau level. These are states in which the macroscopic particle density is uniform. For example a Wigner crystal, while microscopically inhomogeneous, can be considered homogeneous on a macroscopic scale; the average density is uniform. In fact, most states of interest in condensed matter physics have this property.

In general the normalization integral for any wavefunction $\Psi_{0}\left(\left\{\mathbf{r}_{j}\right\}\right)$ that satisfies cylindrical boundary conditions in the lowest Landau level is representable in the form of equation (16), where

$$
W\left(\left\{\mathbf{r}_{j}\right\}\right)=\frac{2 \pi N}{\tau_{2} L^{2}} \sum_{j=1}^{N} y_{j}^{2}+\tilde{U}\left(\left\{\mathbf{r}_{j}\right\}\right),
$$

and $\tilde{U}\left(\left\{\mathbf{r}_{j}\right\}\right)=-v \ln \left|f\left(\left\{\mathbf{r}_{j}\right\}\right)\right|^{2}$ is the interaction energy of the equivalent classical system (here $f\left(\left\{\mathbf{r}_{j}\right\}\right)$ is the analytic factor in the many-body wavefunction, equation (11)). The interpretation of the first term in equation (35) is exactly the same as for the Laughlin states: it is the energy of classical particles in the electrostatic field of a homogeneous charged background with the density $\rho=N /\left(\tau_{2} L^{2}\right)$. Depending on the form of the wavefunction $\Psi_{0}$, the second term can contain both two-particle and multi-particle interactions. In general the form of these interactions is extremely complicated; they are neither translationally nor rotationally invariant. However, if the physical density distribution of the quantum state under consideration is macroscopically homogeneous with a mean density $\bar{n}=N / L^{2}$, then the interaction energy $\tilde{U}\left(\left\{\mathbf{r}_{j}\right\}\right)$ of the equivalent classical system must contain a long-range Coulomb contribution of the form $\sum_{j<k} \tilde{V}\left(\mathbf{r}_{j}, \mathbf{r}_{k}\right)$. The presence of this pairwise logarithmic interaction is mandatory to compensate for the background potential and protect the macroscopic homogeneity of the classical gas. Hence for macroscopically homogeneous states in the lowest Landau level the energy of the equivalent classical plasma should take the form

$$
W\left(\left\{\mathbf{r}_{1}\right\}\right)=\frac{2 \pi N}{\tau_{2} L^{2}} \sum_{j=1}^{N} y_{j}^{2}+\sum_{j<k} \tilde{V}\left(\mathbf{r}_{j}, \mathbf{r}_{k}\right)+\tilde{U}_{\mathrm{sr}}\left(\left\{\mathbf{r}_{j}\right\}\right),
$$

where the last term $\tilde{U}_{\text {sr }}\left(\left\{\mathbf{r}_{j}\right\}\right)$ can contain only short-range (possibly multi-particle, anisotropic, etc) interactions. Since short-range interactions do not spoil the extensive character of the free energy, the chain of arguments that led us from equation (16) to (31) remains valid for any macroscopically homogeneous quantum Hall state. Therefore the Lorentz shear modulus for such states can be calculated as follows

$$
\Lambda_{0}=\frac{\hbar}{2 v}\left[\frac{\partial}{\partial \tau_{2}} P_{\mathrm{int}}\left(\frac{n}{\tau_{2}}\right)\right]_{\tau_{2}=1}=-\frac{\hbar}{2 v} K_{\mathrm{int}}(n),
$$

where $P_{\text {int }}(\rho)$ and $K_{\text {int }}(\rho)$ are the interaction pressure and the isothermal bulk modulus of the corresponding classical plasma. 
As a simple example consider the wavefunction proposed by Wexler and Ciftja [22] for the nematic liquid crystal state at $v=1 / 3$. The cylindrical generalization of this wavefunction contains an analytic factor $f$ the form

$$
\begin{gathered}
f\left(z_{1}, \ldots, z_{N}\right)=\prod_{j<k}\left(\mathrm{e}^{2 \pi \mathrm{i} z_{j} / L}-\mathrm{e}^{2 \pi \mathrm{i} z_{k} / L}\right) \\
\quad \times\left(\mathrm{e}^{2 \pi \mathrm{i}\left(z_{j}-\alpha / 2\right) / L}-\mathrm{e}^{2 \pi \mathrm{i}\left(z_{k}+\alpha / 2\right) / L}\right) \\
\times\left(\mathrm{e}^{2 \pi \mathrm{i}\left(z_{j}+\alpha / 2\right) / L}-\mathrm{e}^{2 \pi \mathrm{i}\left(z_{k}-\alpha / 2\right) / L}\right),
\end{gathered}
$$

where $\alpha$ is a complex number, which we set equal to i $a$, with $a$ real and positive. With this choice the system remains invariant under rotations about the axis of the cylinder. The parameter $a$ is the microscopic distance (of the order of the magnetic length) by which two of the three zeros of the $v=$ 1/3 Laughlin wavefunction are displaced from their 'regular' position on top of the particle. The Laughlin wavefunction is recovered by setting $a=0$. It is now straightforward to verify that the classical system that is equivalent to this wavefunction contains, in addition to the interactions of equation (17), a two-body short-range interaction of the form (in the physically relevant $2 \mathrm{D}$ regime, $\left|z_{i}-z_{j}\right| \ll L$ )

$$
\tilde{U}_{\mathrm{sr}}\left(\left\{\mathbf{r}_{j}\right\}\right)=-\frac{v}{2} \sum_{i \neq j} \ln \left|1+\frac{a^{2}}{\left(z_{i}-z_{j}\right)^{2}}\right|^{2} .
$$

This interaction is strongly anisotropic and exhibits logarithmic singularities at interparticle distance 0 (independent of direction) and $a$ (along the $y$ axis). It is clearly very difficult to calculate the bulk modulus of this system from classical statistical mechanics. However, if we could, by some independent method, calculate the Lorentz shear modulus of the wavefunction (38), then the problem would be solved.

\section{Conclusion}

In this paper we have shown that the calculation of the Lorentz shear modulus in a macroscopically homogeneous electronic system in the lowest Landau level can be mapped to the calculation of the bulk modulus of an equivalent classical system. Application of this approach to Laughlin's fractional quantum Hall states gives a value of the modulus independent of filling factor, in agreement with the result of [10]. We have also suggested that the application of this mapping to non-Laughlin states in the lowest Landau level could reveal interesting connections between the elastic properties of quantum and classical statistical systems.

\section{Acknowledgments}

One of us (GV) gratefully acknowledges support from NSF under grant No. DMR-0705460. The work of IVT was supported by the Ikerbasque Foundation. We thank Nick Read and Yosi Avron for their help in clarifying the numerical factor of equation (2) and for bringing to our attention equation (1).

\section{Appendix. Electrostatics on a cylinder}

In this appendix we calculate a few important ingredients of a classical neutral jellium model on a cylinder.

The interaction potential $V\left(\mathbf{r}-\mathbf{r}^{\prime}\right)$ of two-point unit charges living on a cylindrical surface is a periodic solution of a 2D Poisson equation

$$
\begin{gathered}
\nabla^{2} V(x, y)=-4 \pi \delta\left(x-x^{\prime}+n L\right) \delta\left(y-y^{\prime}\right), \\
V(x+L, y)=V(x, y)
\end{gathered}
$$

where $L$ is the circumference of the cylinder and $n$ is an integer number. This equation is readily solved by the Fourier transformation. The result takes the following form

$$
V\left(\mathbf{r}-\mathbf{r}^{\prime}\right)=-2 \pi \frac{\left|y-y^{\prime}\right|}{L}-\ln \left|1-\mathrm{e}^{2 \pi \mathrm{i} \frac{x-x^{\prime}+\mathrm{i} \mid y-y^{\prime}}{L}}\right|^{2} .
$$

It is easy to see that this potential has correct $1 \mathrm{D}$ and $2 \mathrm{D}$ asymptotic forms

$$
V\left(\mathbf{r}-\mathbf{r}^{\prime}\right)= \begin{cases}-\frac{2 \pi}{L}\left|y-y^{\prime}\right|, & \left|y-y^{\prime}\right| \gg L \\ -\ln \left|\mathbf{r}-\mathbf{r}^{\prime}\right|^{2}, & \left|\mathbf{r}-\mathbf{r}^{\prime}\right| \ll L .\end{cases}
$$

It is also straightforward to check the following useful representation for the potential $V\left(\mathbf{r}-\mathbf{r}^{\prime}\right)$

$$
V\left(\mathbf{r}-\mathbf{r}^{\prime}\right)=-2 \pi \frac{y+y^{\prime}}{L}-\ln \left|\mathrm{e}^{2 \pi \mathrm{i} z / L}-\mathrm{e}^{2 \pi \mathrm{i} z^{\prime} / L}\right|^{2} .
$$

Now we find the interaction potential $U_{\mathrm{pb}}(\mathbf{r})$ of a negatively charged particle with a positive background charge density $n_{\mathrm{b}}(\mathbf{r})$ that is homogeneously distributed on a cylinder and occupies the region $-\frac{L \tau_{2}}{2}<y<\frac{L \tau_{2}}{2}$

$$
n_{\mathrm{b}}(\mathbf{r})=\rho \theta\left(\frac{L \tau_{2}}{2}-|y|\right),
$$

where $\rho=\frac{N}{L L \tau_{2}}$ and $N$ is the total charge of the background. The potential $U_{\mathrm{pb}}(\mathbf{r})$ is given by the following integral

$$
\begin{aligned}
& U_{\mathrm{pb}}(\mathbf{r})=-\int \mathrm{d} \mathbf{r}^{\prime} V\left(\mathbf{r}-\mathbf{r}^{\prime}\right) n_{\mathrm{b}}\left(\mathbf{r}^{\prime}\right)=-\pi \frac{N}{2} \frac{L \tau_{2}}{L} \\
& -2 \pi \rho y^{2}+2 \pi \rho\left(|y|-\frac{L \tau_{2}}{2}\right)^{2} \theta\left(|y|-\frac{L \tau_{2}}{2}\right) .
\end{aligned}
$$

Similarly one calculates the background-background interaction energy

$$
W_{\mathrm{bb}}=\frac{1}{2} \int \mathrm{d} \mathbf{r} \mathrm{d} \mathbf{r}^{\prime} V\left(\mathbf{r}-\mathbf{r}^{\prime}\right) n_{\mathrm{b}}\left(\mathbf{r}^{\prime}\right) n_{\mathrm{b}}(\mathbf{r})=\pi \frac{N^{2}}{3} \frac{L \tau_{2}}{L} .
$$

\section{References}

[1] Vignale G, Ullrich C A and Conti S 1997 Time-dependent density functional theory beyond the adiabatic local density approximation Phys. Rev. Lett. 79 4878-81

[2] Tokatly I V 2005 Quantum many-body dynamics in a Lagrangian frame: II. Geometric formulation of time-dependent density functional theory Phys. Rev. B 71165105 
[3] Tokatly I V 2007 Time-dependent deformation functional theory Phys. Rev. B 75125105

[4] Tokatly I V 2006 Magnetoelasticity theory of incompressible quantum Hall liquids Phys. Rev. B 73205340

[5] Tokatly I V 2006 Unified hydrodynamics theory of the lowest Landau level Phys. Rev. B 74035333

[6] Tokatly I V and Vignale G 2007 New collective mode in the fractional quantum Hall liquid Phys. Rev. Lett. 98026805

[7] Tokatly I V and Vignale G 2007 Lorentz shear modulus of a two-dimensional electron gas at high magnetic field Phys. Rev. B 76 161305(R)

Tokatly I V and Vignale G 2009 Phys. Rev. B 79199903 (erratum)

[8] Landau L D and Lifshitz E M 1986 Course of Theoretical Physics 3 edn, vol 7 Theory of Elasticity (Oxford: Pergamon)

[9] Avron J E, Seiler R and Zograf P G 1995 Viscosity of quantum Hall fluids Phys. Rev. Lett. 75 697-700

[10] Read N 2009 Non-Abelian adiabatic statistics and Hall viscosity in quantum Hall states and $p_{x}+\mathrm{i} p_{y}$ paired superfluids Phys. Rev. B 79045308

[11] Lifshitz E M and Pitaevskii L P 1981 Course of Theoretical Physics vol 10 Physical Kinetics (Oxford: Pergamon)

[12] Lopez A and Fradkin E 1991 Fractional quantum Hall effect and Chern-Simons Gauge theories Phys. Rev. B 44 5246-62

[13] Lopez A and Fradkin E 1993 Response functions and spectrum of collective excitations of fractional-quantum-Hall-effect systems Phys. Rev. B 47 7080-94
[14] Simon S H and Halperin B I 1993 Finite-wavevector electromagnetic response of fractional quantized Hall states Phys. Rev. B 48 17368-87

[15] Heinonen O (ed) 1998 Composite Fermions: a Unified View of the Quantum Hall Effect (Singapore: World Scientific)

[16] Laughlin R B 1983 Anomalous quantum Hall effect: an incompressible quantum fluid with fractionally charged excitations Phys. Rev. Lett. 50 1395-8

[17] Lévay P 1995 Berry phases for Landau Hamiltonians on deformed tori J. Math. Phys. 36 2792-802

[18] Giuliani G F and Vignale G 2005 Quantum Theory of the Electron Liquid (Cambrige: Cambridge University Press)

[19] Thouless D J 1984 Theory of the quantized Hall effect Surf. Sci. 142 147-54

[20] Ciftja O and Wexler C 2003 Monte Carlo simulation method for Laughlin-like states in a disk geometry Phys. Rev. B 67075304

[21] Caillol J M, Levesque D, Weis J J and Hansen J P 1982 A Monte Carlo study of the classical two-dimensional one-component plasma J. Stat. Phys. 28325

[22] Wexler C and Ciftja O 2002 Liquid crystalline states in quantum Hall systems J. Phys.: Condens. Matter 143705

[23] Aschcroft N W and David Mermin N 1976 Solid State Physics (Fortworth, TX: Harcourt Brace College Publishers)

[24] Haldane F D M and Rezayi E H 1985 Periodic Laughlin-Jastrow wavefunctions for the fractional quantized Hall effect Phys. Rev. B 31 2529-31 